\title{
Development of vaccine that could save lives of children in the North at a standstill
}

\author{
C Cite as: CMAJ 2019 March 11;191:E293-4. doi: 10.1503/cmaj.109-5715
}

Posted on cmajnews.com on Feb. 20, 2019.

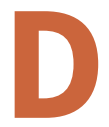

r. Yoko Schreiber, an infectious disease specialist in the Northwest Ontario city of Sioux Lookout, paid close attention when a family physician presented rounds on two babies with meningitis. The babies, from the same First Nations community, got sick several years apart, but the same bacteria grew from their blood cultures: Haemophilus influenza type a (Hia). The bacteria causes potentially deadly infections, such as meningitis and pneumonia, that affect Indigenous children across the North. Most doctors are more familiar with Hia's bacterial cousin, Haemophilus influenza type b (Hib), the culprit in many childhood deaths and disabilities until a vaccine all but wiped it out in the early 1990s.

Schreiber, however, knew about Hia because she's involved in a project to develop a vaccine against it. The project has federal support but has sparked debate. Some doctors have put all their focus on the vaccine, but others are concerned about the absence of input from Indigenous people and the lack of plans to address the many other factors contributing to disease in the North.

The initial push for a vaccine came from a senior scientist with the Public Health Agency of Canada (PHAC). Recent PHAC data show that, in Nunavut, most of those with Hia are Inuit children aged four or younger and the fatality rate is $9.1 \%$. In Nunavik, an Indigenous area of Northern Quebec, an outbreak in a few villages in 2013 sickened 10 children and took the lives of two infants.

Dr. Holden Sheffield, a pediatrician at Iqaluit's Qikiqtani General Hospital, said the fact that Hia causes invasive disease with substantial morbidity suggests "it would be obviously beneficial in a lot of

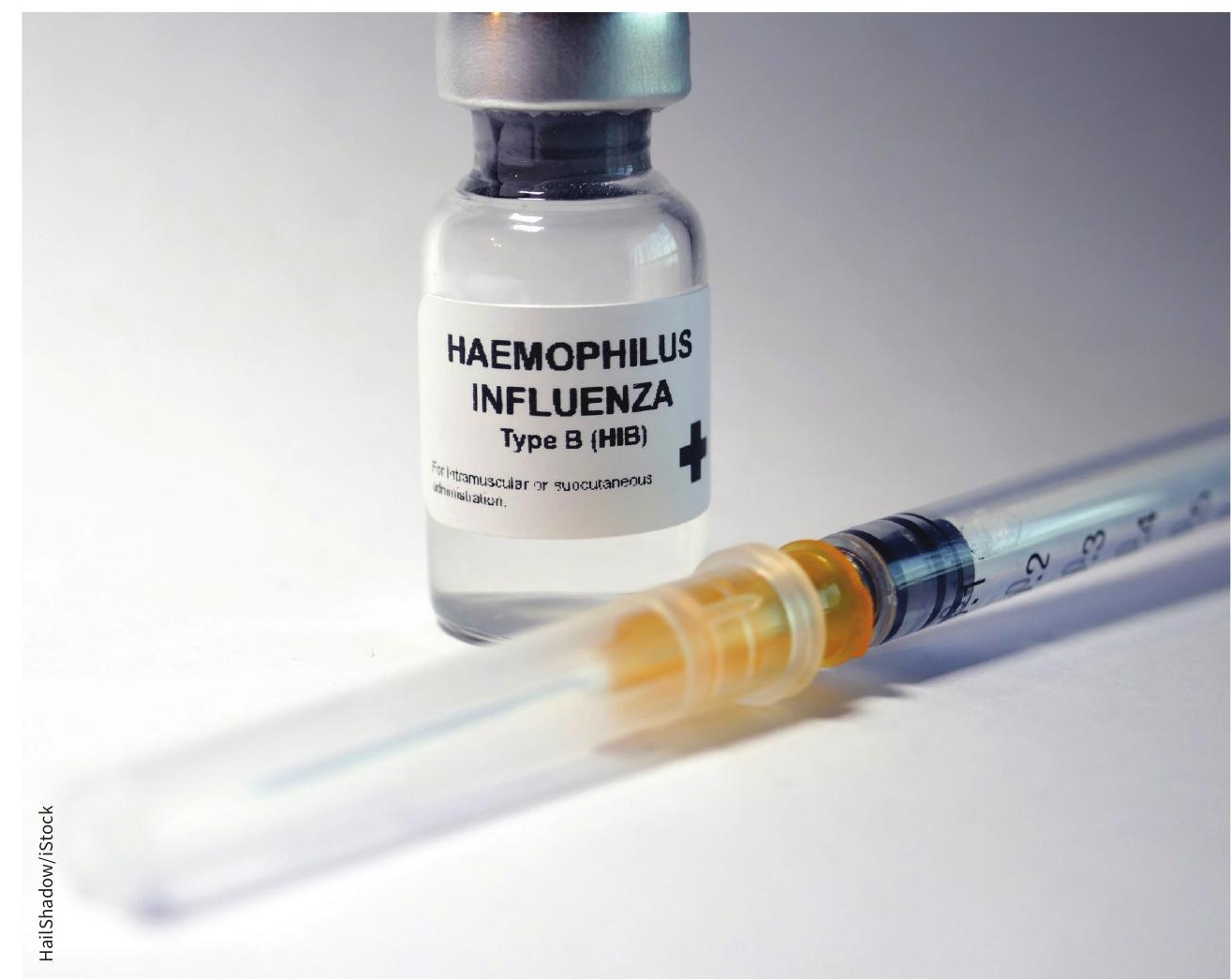

A vaccine for Haemophilus influenzae type b prevented many deaths, but there is still no vaccine for its potentially deadly cousin, Haemophilus influenzae type a.

ways to vaccinate for this." Vulnerable populations in Canada and the United States should be targeted with a vaccine "sooner rather than later," suggested Ian Plumb, an epidemiologist with the US Centers for Disease Control and Prevention.

PHAC put Hia first on its list of human vaccines to be developed, and the National Research Council of Canada (NRC) made it the pilot project of an initiative intended to jumpstart Canada's vaccine industry. In 2015, those efforts resulted in a vaccine prototype. Two years later, the NRC licensed the technology to a start-up called InventVacc Biologicals to turn it into an experimental vaccine and test it in clinical trials. But in Schreiber's view, something crucial is missing.

"There are all these conversations going on about this vaccine development and they don't know how to engage communities," she said. Ideally, affected communities would be giving their perspectives on the infection and the vaccine, but that's not happening. Most people in Indigenous communities have never heard of Hia, she said.

Kristin Burnett, an associate professor of Indigenous learning at Lakehead University, who's been conducting 
interviews about Hia in Northern Ontario, says that people are not opposed to having the vaccine in their communities, but when she mentions clinical trials, they "shut down." So, she plans to create a toolkit that communities can use to know their rights if they become involved in medical research.

But even if community members are on board, targeting a single bacterial infection in isolation is a short-sighted approach, according to Dr. Mike Kirlew, a family physician in Sioux Lookout. Other factors should also be considered: overcrowded housing, poor indoor air quality, limited access to clean water, and other problems that contribute to infectious diseases in the North.

"You can't just apply a vaccination and expect it to take away the problem," he said. "You need to use that as part of a larger strategy."

The vaccine may not be available for some time anyway. InventVacc hasn't submitted the product to Health Canada for approval for use in humans. Dr. Luis Barreto, a scientific advisor with the company, said InventVacc won't take the final steps toward that goal until it obtains funding for clinical trials. But no one involved in the project can say where those funds would come from.

PHAC's technology for an Ebola vaccine sat on a shelf for years and was turned into a viable vaccine only after thousands lost their lives in an outbreak of the disease in West Africa. Hia, however, won't cause a huge outbreak. "That's not the way that organism works," said Dr. Scott Halperin, director of the Canadian Centre for Vaccinology. "So, the potential is, something like this could sit on the shelf for a whole lot longer."

Miriam Shuchman, Toronto, Ont. 